\title{
1 Regulation of plasmodesmata at specific cell-cell interfaces
}

2 Zhongpeng Li and Kyaw Aung*

3 Department of Genetics, Development, and Cell Biology, Iowa State University, Ames, IA

4 50011, USA

5 * Correspondence:

6 Corresponding Author

7 kaung@iastate.edu

\section{Abstract}

Precise exchange of information and resources among cells is essential for multicellular organisms.

11 Intercellular communication among diverse cell types requires differential mechanisms to achieve

12 the specific regulation. Despite the significance of intercellular communication, it is largely

13 unknown how the communication between different cells is regulated. Here, we report that two

14 members of plasmodesmata-located proteins modulate plasmodesmata at two distinct cell-cell

15 interfaces.

\section{Main}

18 Plasmodesmata (PD) are membrane-lined channels connecting adjoining plant cells, allowing the exchange of signals and resources (Lucas et al., 2009). The PD-dependent communication among cells is fundamental for developmental regulation and stress responses in plants (Sager and Lee,

21 2018). PD allow translocating photosynthetic products from mature leaves (source tissues) to non-

22 photosynthetic parts of the plant (sink tissues; Comtet et al., 2017; Liesche and Patrick, 2017).

23 Recent findings also highlighted the crucial role of PD in plant immunity against filamentous and

24 bacterial pathogens (Lee et al., 2011; Faulkner et al., 2013; Cao et al., 2015; Aung et al., 2020;

25 Tomczynska et al., 2020). The function of PD is controlled by the homeostasis of a plant

26 polysaccharide, callose. Callose synthases (CalSs) and $\beta-1,3$ glucanases mediate the biosynthesis

27 and degradation of callose, respectively, at the plasma membrane of PD (De Storme and Geelen,

28 2014). Callose deposition at PD suppresses the PD-dependent movement of molecules between

29 adjoining cells, presumably by narrowing the PD aperture. Other than callose, plasmodesmata-

30 located proteins (PDLPs) also play important roles in regulating the plasmodesmal function, but

31 how PDLPs regulate PD function is unclear (Lee et al., 2011; Wang et al., 2020). 
33 To better understand the function of PDLPs, we individually overexpressed all eight members of 34 PDLP (PDLP1-8; Thomas et al., 2008) in Arabidopsis wild-type Col-0. The PDLPs were fused with His and Flag (HF) tag. The levels of protein expression in the transgenic plants were assessed by immunoblot analyses using a Flag antibody (Figure 1a and Supplemental Figure 1a). Here, we report the characterization of $p 35 S:: P D L P 5-H F$ and $p 35 S:: P D L P 6-H F$. Consistent with a previous report (Lee et al., 2011), a stunted growth phenotype was observed for the $p 35 S:: P D L P 5-H F$ lines compared to a vector control $p 35 S:: H F-Y F P$. We also observed a positive correlation between the expression level of PDLP5 and the growth phenotype (Supplemental Figure 1a-1b; Lee et al., 2011). In addition, we identified p35S::PDLP6-HF transgenic lines with more extreme developmental phenotypes, including a stunted growth and late flowering. Similar to PDLP5, transgenic plants with higher expression level of PDLP6 exhibit a more severe dwarfism in plant growth (Figure 1a-1b and Supplemental Figure 1c). We hypothesized that the overexpression of PDLP5 and PDLP6 affects the PD-dependent sugar translocation for the following reasons: (1) PD are required for symplastic movement of sugars between mesophyll cells in mature leaves (Schulz et al., 2015), (2) PD are involved in sugar loading within phloem in Arabidopsis (Liesche et al., 2017), and (3) Arabidopsis mutants compromised in sugar translocation exhibit a stunted growth phenotype (Srivastava et al., 2008; Chen et al., 2012).

In mature leaves, chloroplasts within mesophyll cells (MCs) convert solar energy into chemical energy, producing sugars. The carbohydrate can be stored temporarily in the chloroplasts as starch or translocated to different parts of the plants. To translocate photosynthetic products, sugars move from MCs to sieve elements (SEs) through different pathways. It's generally believed that

55 photosynthetic products move symplastically through PD between photosynthetic cells, including MCs and bundle sheath cells (BSs), and are loaded into phloem for long-distance transport (Lucas

57 et al., 2013). In Arabidopsis, three major cell types make up phloem: phloem parenchyma cells 58 (PPs), companion cells (CCs), and SEs. Within phloem, symplastic and apoplastic phloem loading 59 have been reported for different plant species. Symplastic phloem loading moves sugars mainly 60 through PD connecting different phloem cell types. Arabidopsis utilizes apoplastic phloem 61 loading, in which sugars are exported from PPs into the extracellular space and imported into CCs. 62 From CCs, sugars move symplastically into SEs for long-distance transport. Arabidopsis 

(SWEET11 and SWEET12) function as sugar exporters on the plasma membrane of PPs (Chen et al., 2012), whereas a sucrose-proton symporter 2 (SUC2) protein imports sugars from the apoplast into CCs in Arabidopsis (Srivastava et al., 2008). sweet 11;12 and suc2 Arabidopsis mutants exhibit compromised sugar translocation from source to sink, resulting in overaccumulation of starch in mature leaves and stunted growth (Srivastava et al., 2008; Chen et al., 2012). Sugars from CCs move into SEs through PD (Lucas et al., 2013; Liesche and Patrick, 2017), completing the sugarloading process. The enucleated SEs transport sugars to other parts of the plants (Oparka and

71 Turgeon, 1999; Chen et al., 2015; Zhang and Turgeon, 2018). In a simplified pathway, sugars move from $\mathrm{MCs} \rightarrow \mathrm{BSs} \rightarrow \mathrm{PPs} \rightarrow \mathrm{CCs} \rightarrow$ SEs in mature leaves to translocate sugars in Arabidopsis.

To investigate whether the stunted growth phenotypes of the transgenic plants are due to defects in symplastic translocation of sugar in mature leaves, we first examined starch accumulation in p35S::PDLP6-HF transgenic plants. The aerial portions of 3-week-old plants were stained with Lugol's solution at the end of the night, when overall starch accumulation in mature leaves is at its lowest (Chen et al., 2012). When grown under a light intensity used for standard Arabidopsis growth $\left(110 \mu \mathrm{mol} \mathrm{m} \mathrm{m}^{-2} \mathrm{~s}^{-1}\right), 3$ out of 4 p35S::PDLP6-HF independent transgenic plants overaccumulate starch in mature rosette leaves, exhibiting a dark blue color, compared to p35S::HF-YFP. Similar to plant growth phenotype, transgenic plants with higher expression level of PDLP6 contain more starch in their mature leaves (Figure 1c). For further analyses, we choose p35S::PDLP6-HF (\#2). We also determined starch accumulation phenotype in p35S::PDLP5-HF (\#1) due to its stunted growth phenotype (Supplemental Figure 1). To compare starch accumulation phenotype with mutants defective in sugar translocation, we included Arabidopsis mutants, cher1-4 (Kraner et al., 2017) and sweet11;12 (Chen et al., 2012) as both mutants overaccumulate starch in leaves. Consistent with previous report (Kraner et al., 2017), cher1-4 shows starch overaccumulation in mature rosette leaves. Col-0, p35S::HF-YFP, sweet11;12, and p35S::PDLP5-HF all display comparable starch accumulation phenotype (Supplemental Figure 2a). As sweet 1,12 exhibits starch accumulation only when the plants were grown under a high

91 light condition (Chen et al., 2012), we irradiated the mutants and transgenic plants with a high

92 light intensity $\left(200 \mu \mathrm{mol} \mathrm{m} \mathrm{m}^{-2} \mathrm{~s}^{-1}\right)$ for a week. The starch overaccumulation phenotype became 93 apparent in sweet 11;12 as well as p35S::PDLP5-HF, when the plants were grown under a high 
94 light condition (Figure 1c and Supplemental Figure 2b). Intriguingly, we observed distinct starch 95 accumulation patterns among the genotypes. p35S::PDLP6-HF and sweet 11;12 accumulates starch evenly in mature leaves, whereas $p 35 S:: P D L P 5-H F$ and cher 1-4 lack starch accumulation in and around vascular tissues in mature leaves (Figure 1d). As SWEET11 and SWEET12 specifically express at PPs within phloem (Chen et al., 2012), sugars are likely still able to move from MCs to PPs in the sweet 1,12 mutant. cher1-4, on the other hand, has a reduced number of PD between mesophyll cells to allow for symplastic movement of sugars (Kraner et al., 2017); consequently, it is possible that most sugars are trapped within the photosynthetic cells of the mutant. To determine cell type-specific starch accumulation in the mutants and transgenic plants, leaf discs from high light-treated plants were subjected to histological sectioning and starch staining. Periodic acid/Schiff (PAS) reagent labels polysaccharide in cell wall and starch grains in chloroplasts. Consistent with whole tissue staining (Figure 1d), cher1-4, sweet11;12, p35S::PDLP5-HF, and p35S::PDLP6-HF exhibit darker PAS-stained starch grains in chloroplasts of mesophyll cells compared to that of Col-0 and $p 35 S: \because H F-Y F P$. In line with our prediction, cher1-4 and p35S::PDLP5-HF contain fewer starch grains in BSs, whereas sweet11;12 and p35S::PDLP6-HF contain many starch grains in their BSs. We also observed starch grains in PPs of sweet11;12 (Figure 1e). Together, the findings support that the sugar translocation is likely blocked between (1) MC-MC and MC-BS in cher1-4 and p35S::PDLP5-HF, (2) BS-PP in p35S::PDLP6-HF, and (3) PP-CC in sweet11;12. The distinct patterns of starch accumulation between $p 35 S:: P D L P 5-H F$ and $p 35 S:: P D L P 6-H F$ suggest that PDLP5 and PDLP6 express in and function at specific cell types to regulate the plasmodesmal function at two distinct cell-cell

To determine whether PDLP5 and PDLP6 express in a cell-type-specific manner, we generated native promoter driven $p P D L P 5:: P D L P 5-Y F P$ and $p P D L P 6:: P D L P 6-Y F P$ transgenic Arabidopsis plants. $T_{3}$ generations of the transgenic plants were subjected to confocal imaging. The expression of PDLP5-YFP was mainly detected between epidermal cells, whereas no PDLP5-YFP signals

121 were observed in mesophyll cells and leaf vasculature. PDLP6-YFP, on the other hand, was 122 expressed specifically in leaf vasculature (Figure 2a). We postulate that PDLP6-YFP expresses 123 exclusively in PP as PDLP6 transcripts were detected predominantly in the cell type (Kim et al., 
125

126

127

128

129

was detected in epidermis and cortex (Figure 2a). Transgenic plants co-expressing PDLP-YFP and cell type-specific markers tagged with a compatible fluorescent protein (e.g., red fluorescent protein) for simultaneous imaging will further confirm the cell type-specific expression of PDLP6 in phloem. In parallel, we determined the promoter activity of PDLP5 and PDLP6 using $\beta$ Glucuronidase (GUS) reporter gene assay. Two transgenic lines, pPDLP5::GUS and pPDLP6::GUS, were subjected to GUS activity staining as previously described (Li et al., 2016). Among 20 independent pPDLP5::GUS transgenic plants, 15 of them exhibit a much higher GUS activity in leaf epidermal cells. The majority (13 out of 19) of pPDLP6::GUS transgenic plants displays the highest activity in leaf vasculature (Supplemental Figure 3). Representative transgenic lines of $p P D L P 5:: G U S$ and pPDLP6::GUS were selected to analyze GUS activity in roots. pPDLP5::GUS transgenic plants exhibit GUS activity in epidermis and cortex, but not in the vasculature. $p P D L P 6:: G U S$ transgenic plants, on the other hand, exhibit the highest GUS activity in the vasculature, likely phloem (Supplemental Figure 3). Together, our findings demonstrate that PDLP5 and PDLP6 are expressed in distinct and non-overlapping cell types. Interestingly, PDLP5 was reported to express in lateral root primordium-overlaying cells during lateral root emergence process (Sager et al., 2020). Further investigation will reveal the cell type-specific expression and function of PDLPs at different developmental stages and tissues.

While the molecular mechanism underlying PDLP function is unclear, the expression level of PDLP5 is highly associated with callose accumulation at PD in plants (Lee et al., 2011; Li et al., 2020). Similarly, the expression of PDLP1 is required for callose accumulation at haustoria during downy mildew infection in Arabidopsis (Caillaud et al., 2014). To further investigate the cell typespecific roles of PDLP5 and PDLP6, we examined whether the overexpression of PDLP5 or PDLP6 leads to the overaccumulation of callose at specific cell-cell interfaces. To detect callose accumulation, $p 35 S:: P D L P 5-H F, p 35 S:: P D L P 6-H F$, and $p 35 S:: H F-Y F P$ leaves were stained with aniline blue. Consistent with the previous report (Lee et al., 2011), we observed a higher accumulation of callose between epidermal cells in p35S::PDLP5-HF as compared to both p35S::PDLP6-HF and $p 35 S: \because H F-Y F P$. We also detected a higher callose level at PD connecting mesophyll cells in p35S::PDLP5-HF (Figure 2b-2c). As PDLP6 expresses specifically in vasculature, we also examined the callose accumulation between vascular cells. To this end, roots of the transgenic plants were stained with aniline blue and imaged with confocal microscopy due 
to their tissue transparency. We observed a much higher accumulation of callose in epidermis and cortex of $p 35 S:: P D L P 5-H F$ than $p 35 S:: H F-Y F P$ and $p 35 S:: P D L P 6-H F$. In contrast, p35S::PDLP6-HF exhibits the highest accumulation of callose in vascular cells (Figure 2d). Fluorescence intensity profiles were consistent among data collected from 25 individual transgenic plants for each genotype (Supplemental Figure 4). These findings support that the overexpression of PDLP5 and PDLP6 leads to the higher accumulation of callose at specific cell-cell interfaces.

We observed the cell type-specific functions of PDLP5 and PDLP6 while examining the transgenic plants overexpressing PDLP5 or PDLP6 genes. When driven by the same $35 S$ constitutive promoter, PDLP5-YFP and PDLP6-YFP are expressed in most cell types (Supplemental Figure 5; Aung et al., 2020), suggesting that PDLP5-HF and PDLP6-HF express ubiquitously in the transgenic plants. From the starch and callose accumulation phenotypes, it is clear that the $35 S$ driven expression of PDLP6 alters the plasmodesmal function only in the cell types where it is natively expressed. This suggests that different members of PDLPs regulate PD in a cell typespecific manner. One possibility for why constitutive expression of PDLP6 does not lead to PD defects in all cell types is that PDLPs might require other cell type-specific proteins to regulate PD. This hypothesis is supported by the finding that PDLP5 functions together with CalS8 to maintain basal callose homeostasis at PD, whereas it works together with CalS1 to deposit callose at PD during salicylic acid-dependent plasmodesmata regulation in Arabidopsis (Cui and Lee, 2016). The findings imply that different CalSs might function together with different PDLPs to regulate callose accumulation at PD in specific cell types. Alternatively, other unknown cell typespecific partners or factors might contribute to the PDLPs-dependent function in unique cell types. Although PDLP5-YFP is not detected in mesophyll cells when driven under the native promoter, the overexpression of PDLP5-HF leads to callose overaccumulation between mesophyll cells (Figure 2b-2c) and likely blocks sugars movement between photosynthetic cells. It's plausible that the expression of PDLP5-YFP is under detectable threshold using confocal microscopy. Alternatively, functional partners of PDLP5 might be present in mesophyll cells, modulating callose accumulation and the plasmodesmal function.

The findings in this article begin to reveal how communication among distinct cell types is regulated in multicellular organisms. Further research should uncover the function of PDLPs and 
their partner proteins in regulating PD at the molecular level. As PD are critical in plant growth and defense, understanding the cell type-specific regulation of PD will provide a valuable toolkit to improve crop production.

\section{Methods}

\section{Plant Material, Growth Conditions, Transformation, and Plant Selection}

Arabidopsis (Arabidopsis thaliana) plants were grown at $22^{\circ} \mathrm{C}$ with $50 \%$ humidity and irradiated with $110 \mu \mathrm{mol} \mathrm{m}^{-2} \mathrm{~s}^{-1}$ white light for $14 \mathrm{~h}$ per day. To grow plants under a higher light intensity, plants were irradiated with $200 \mu \mathrm{mol} \mathrm{m} \mathrm{m}^{-2} \mathrm{~s}^{-1}$ white light. Transgenic Arabidopsis plants were generated using the simplified transformation method (https://plantpath.wisc.edu/simplifiedarabidopsis-transformation-protocol/). To select for transgenic plants harboring transgenes containing the hygromycin-resistance gene, $\mathrm{T}_{1}$ seeds were selected on $0.5 \times \mathrm{LS}$ medium containing $25 \mu \mathrm{g} / \mathrm{mL}$ hygromycin. For those harboring transgenes containing the glufosinate-resistance gene, $\mathrm{T}_{1}$ seeds were germinated on soil and 1-week-old seedlings were sprayed with $0.1 \%(\mathrm{v} / \mathrm{v})$ Finale Herbicide (Bayer) and 0.05\% (v/v) Silwet L-77 (PhytoTech). The $\mathrm{T}_{2}$ or $\mathrm{T}_{3}$ plants were selected on $0.5 \times \mathrm{LS}$ medium containing $10 \mu \mathrm{g} / \mathrm{mL}$ glufosinate-ammonium.

\section{Gene Cloning and Plasmid Construction}

205 The coding sequences of PDLP1-8 were amplified with Gateway-compatible primers from cDNA synthesized from total RNA extracted from wild-type (Col-0) seedlings, and the coding sequence of yellow fluorescent protein (YFP) from pEarleyGate 101 (Earley et al., 2006) using Phusion High-Fidelity DNA polymerase (ThermoFisher). To generate $p 35 S:: P D L P-H F$ or $p 35 S:: H F-Y F P$, the PCR fragments were cloned into the pDONR 207 entry vector, followed by the pB7-HFC or pB7-HFN destination vector (Lee et al., 2018) using a standard Gateway cloning system (Invitrogen). To generate $p P D L P 5:: P D L P 5-Y F P$ and $p P D L P 6:: P D L P 6-Y F P$ constructs, a 3029 and $3331 \mathrm{bp}$ including the promoter and PDLP genomic fragment with all exons and introns without stop codons were amplified from genomic DNA of wild-type Col-0 using Gateway-

215 by the pGWB540 destination vector (Nakagawa et al., 2007) using a standard Gateway cloning system (Invitrogen). To generate $p P D L P 5:: G U S$ and $p P D L P 6:: G U S$ constructs, the promoters (2051 bp and 1505 bp for PDLP5 and PDLP6, respectively) were amplified from genomic DNA 
218

219

220

221

222

223

224

225

226

227

228

229

230

231

232

233

234

235

236

237

238

239

240

241

242

243

244

245

246

247

248

of wild-type Col-0 using Gateway-compatible primers. The PCR fragments were cloned into the pDONR 207 entry vector, followed by the pMDC163 destination vector (Curtis and Grossniklaus, 2003) using a standard Gateway cloning system (Invitrogen). All primers used for cloning are listed in Supplemental Table 1.

\section{Whole Tissue Starch Staining}

The aerial portion of 4-week-old Arabidopsis plants grown under regular light were harvested at the end of the night. For high light-treated plants, 4-week-old Arabidopsis plants grown under regular light were moved into a growth chamber with the same growth conditions except a higher light intensity. The inflorescence was removed and the rosette leaves were decolored overnight with $95 \%$ ethanol. The samples were then washed with $\mathrm{ddH}_{2} \mathrm{O}$ and stained with Lugol's solution (Sigma-Aldrich) for 10 minutes and rinsed with $\mathrm{ddH}_{2} \mathrm{O}$. The images were captured with a Canon camera 2 hours after staining.

\section{Tissue Sectioning and Starch Staining}

Leaf punch samples were collected and fixed in FAA fixative (5\% formaldehyde, $5 \%$ glacial acetic acid, 50\% ethyl alcohol). Samples were dehydrated through graded ethanol series (70, 85, 95, $100 \%$ ) for 3-6 hours each concentration. Samples were infiltrated into LR White hard grade resin (Electron Microscopy Sciences) and polymerized at $55^{\circ} \mathrm{C}$ for 48 hours. Sections were made using a Leica UC6 ultramicrotome at $1.5 \mu \mathrm{m}$ thickness. Sections were stained for non-soluble polysaccharides as the following: slides with sections were immersed in periodic acid for 5 minutes, rinsed in distilled water for 5 minutes, stained in Schiff's reagent (Electron Microscopy Sciences) for 10 minutes, rinsed in running tap water for 5 minutes, and air dried. Dry slides were coverslipped using Permount mounting media (Fisher Scientific).

\section{Aniline Blue Staining}

Mature leaves from 4-week-old Arabidopsis leaves were infiltrated with $0.1 \mathrm{mg} / \mathrm{ml}$ aniline blue in 1x PBS buffer ( $\mathrm{pH}$ 7.4). Samples were imaged at 5 minutes after the dye infiltration using confocal microscopy. Ten-day-old seedlings were vacuum-infiltrated with $0.1 \mathrm{mg} / \mathrm{ml}$ of aniline blue in $1 \mathrm{x}$ PBS buffer ( $\mathrm{pH}$ 7.4). Stained root tissues were imaged using confocal microscopy. Callose in mature leaves was quantified using FIJI. For epidermis, images were converted from lsm to tiff. 
8-bit images were used for analysis. Black and white images highlighting callose were created by Auto Threshold which was set by RenyiEntropy white method. Particle Analysis tool was used to outline callose with size from 0.10 to $20 \mu \mathrm{m}^{2}$ and circularity from 0.15 to 1.00 . Quantitative numerical values in $\mu \mathrm{m}^{2}$ were then exported. For mesophyll cells, aniline blue-stained callose area

253 was manually selected and the signal intensity was determined by measuring integrated density.

254 Semi-quantitative evaluation of the relative level of aniline blue-stained callose in the root tissues of the transgenic plants were performed using FIJI. Images were converted from lsm to tiff. 16 bit images were used for analysis. Horizontal lines were drawn near the bottom of the images crossing all root cell types as shown in Figure 2d. Plot profile was used to generate a two-dimensional graph. Value on y-axis represents the relative signal intensity as arbitrary unit (AU).

\section{GUS Activity Staining}

261 GUS staining was performed as previously described (Li et al., 2016) with minor modifications.

262 Mature leaves and small seedlings were immersed in GUS solution (100 mM sodium phosphate 263 buffer [pH 7.0], $10 \mathrm{mM} \mathrm{Na}_{2} \mathrm{EDTA}_{1} 1 \mathrm{mM} \mathrm{K}_{3}\left[\mathrm{Fe}(\mathrm{CN})_{6}\right], 1 \mathrm{mM} \mathrm{K} 4\left[\mathrm{Fe}(\mathrm{CN})_{6}\right], 0.1 \%$ Triton X-100, 264 and $1 \mathrm{mM} \mathrm{X-Gluc).} \mathrm{Samples} \mathrm{were} \mathrm{vacuumed} \mathrm{for} \mathrm{10-40} \mathrm{min,} \mathrm{followed} \mathrm{by} \mathrm{incubation} \mathrm{in} \mathrm{darkness}$ 265 at $37^{\circ} \mathrm{C}$ for $2-16 \mathrm{~h}$. After staining, samples were de-stained in $75 \%$ ethanol. Images were taken 266 using ZEISS Axio Observer.

\section{Confocal Imaging}

269 All confocal images were captured with a confocal laser-scanning microscope (Zeiss LSM 700).

270 A small piece of tissue was mounted with water on a glass slide. For leaf tissues, the abaxial side 271 was imaged. YFP was excited at $514 \mathrm{~nm}$ and emission was collected over the range of 510-550

$272 \mathrm{~nm}$ using SP555. Aniline blue-stained callose was excited at $405 \mathrm{~nm}$ and emission was collected 273 over the range of 420-480 nm using SP555.

\section{Immunoblot Analyses}

276 Arabidopsis leaves were frozen with liquid nitrogen and homogenized with $1600 \mathrm{miniG}$ (SPEX).

277 Protein extraction buffer (60 mM Tris-HCl [pH 8.8], 2\% [v/v] glycerol, $0.13 \mathrm{mM}$ EDTA [pH 8.0],

278 and $1 \times$ protease inhibitor cocktail complete from Roche) was added to the homogenized tissues $279(100 \mu \mathrm{l} / 10 \mathrm{mg})$. The samples were vortexed for $30 \mathrm{~s}$, heated at $70^{\circ} \mathrm{C}$ for $10 \mathrm{~min}$, and centrifuged 
at $13,000 \mathrm{~g}$ for $5 \mathrm{~min}$ at room temperature. The supernatants were then transferred to new tubes.

281 For SDS-PAGE analysis, $10 \mu \mathrm{l}$ of the extract in 1x Laemmli sample buffer (Bio-Rad) was separated on 4-15\% Mini-PROTEAN TGX precast protein gel (Bio-Rad). The separated proteins were transferred to a polyvinylidene fluoride membrane (Bio-Rad) using a Trans-Blot Turbo

284 Transfer System RTA transfer kit following the manufacturer's instructions (Bio-Rad). The membrane was incubated in a blocking buffer $(3 \%$ [v/v] BSA, $50 \mathrm{mM}$ Tris base, $150 \mathrm{mM} \mathrm{NaCl}$, $0.05 \%[\mathrm{v} / \mathrm{v}]$ Tween $20[\mathrm{pH} 8.0])$ at room temperature for $1 \mathrm{~h}$, then incubated overnight with a 1:10,000 dilution of an $\alpha$-Flag-HRP antibody (Sigma-Aldrich catalog No. A8592) at $4^{\circ} \mathrm{C}$. The membrane was washed four times with $1 \times$ TBST $(50 \mathrm{mM}$ Tris base, $150 \mathrm{mM} \mathrm{NaCl}, 0.05 \%$ [v/v] Tween 20 [pH 8.0]) for 10 min. The signals were visualized with SuperSignal West Dura Extended Duration Substrate (Pierce Biotechnology).

\section{Acknowledgements}

293 We thank the ABRC for providing the T-DNA insertion mutants and pMDC163 vector. We thank 294 Tracey Stewart from Roy J. Carver high resolution microscopy facility at Iowa State University 295 (ISU) for helping with histological sectioning and starch staining. We thank the Aung lab members 296 Dr. Yani Chen, Dr. Su-Ling Liu, and Haris Variz. We also thank Dr. Yanhai Yin from ISU, Dr. 297 Michelle Guo from ISU, Dr. Anne Rea from Michigan State University and Dr. Yu-Ti Cheng 298 from Duke university for critically reading the manuscript. This work was supported by the 299 National institute of General Medical Science (Grant R00GM115766 to K.A.).

\section{Contributions}

302 K.A. and Z.P.L. designed the research. Z.P.L. conducted most experiments except the generation of $p 35 S:: P D L P-H F$ and $p 35 S:: P D L P-Y F P$ transgenic lines. K.A. wrote the manuscript with inputs 304 from Z.P.L. 
References:

Aung, K., Kim, P., Li, Z., Joe, A., Kvitko, B., Alfano, J.R., and He, S.Y. (2020). Pathogenic Bacteria Target Plant Plasmodesmata to Colonize and Invade Surrounding Tissues. Plant Cell 32, 595-611.

Caillaud, M.C., Wirthmueller, L., Sklenar, J., Findlay, K., Piquerez, S.J., Jones, A.M., Robatzek, S., Jones, J.D., and Faulkner, C. (2014). The Plasmodesmal Protein PDLP1 Localises to Haustoria-Associated Membranes during Downy Mildew Infection and Regulates Callose Deposition. PLoS pathogens 10, e1004496.

Cao, L., Blekemolen, M.C., Tintor, N., Cornelissen, B.J.C., and Takken, F.L.W. (2018). The Fusarium oxysporum Avr2-Six5 Effector Pair Alters Plasmodesmatal Exclusion Selectivity to Facilitate Cell-to-Cell Movement of Avr2. Mol. Plant 11, 691-705.

Chen, L.Q., Cheung, L.S., Feng, L., Tanner, W., and Frommer, W.B. (2015). Transport of sugars. Annu. Rev. Biochem. 84, 865-894.

Chen, L.Q., Qu, X.Q., Hou, B.H., Sosso, D., Osorio, S., Fernie, A.R., and Frommer, W.B. (2012). Sucrose efflux mediated by SWEET proteins as a key step for phloem transport. Science 335, 207-211.

Comtet, J., Turgeon, R., and Stroock, A.D. (2017). Phloem Loading through Plasmodesmata: A Biophysical Analysis. Plant Physiol. 175, 904-915.

Cui, W., and Lee, J.Y. (2016). Arabidopsis callose synthases CalS1/8 regulate plasmodesmal permeability during stress. Nature Plants 2, 16034.

Curtis M.D. and Grossniklaus U. (2003) A gateway cloning vector set for high-throughput functional analysis of genes in planta. Plant Physiol. 133, 462-469.

De Storme, N., and Geelen, D. (2014). Callose homeostasis at plasmodesmata: molecular regulators and developmental relevance. Front. Plant Sci. 5, 138.

Earley K.W., Hagg J.R., Pontes O., Opper K., Juehne T., Song K., and Pikaard C.S. (2006). Gateway-compatible vectors for plant functional genoics and proteomics. Plant J. 45, 616629.

Faulkner, C., Petutschnig, E., Benitez-Alfonso, Y., Beck, M., Robatzek, S., Lipka, V., and Maule, A.J. 2013. LYM2-dependent chitin perception limits moleculer flux via plasmodesmata. Proc. Natl. Acad. Sci. USA 110, 9166-9170.

Kim J.Y., Symeonidi E., Pang T.Y., Denyer T., Weidauer D., Bezrutczyk M., Miras M., Zöllner N., Wudick M.M., Lercher M., Chen L.Q., Timmermans M.C.P., and Frommer W.B. (2021) Distinct identities of leaf phloem cells revealed by single cell transcriptomics. Plant Cell, koaa060, https://doi.org/10.1093/plcell/koaa060.

Kraner, M.E., Link, K., Melzer, M., Ekici, A.B., Uebe, S., Tarazona, P., Feussner, I., Hofmann, J., and Sonnewald, U. (2017). Choline transporter-like1 (CHER1) is crucial for plasmodesmata maturation in Arabidopsis thaliana. Plant J. 89, 394-406.

Lee C.M., Feke A., Li M.W., Adamchek C., Webb K., Pruneda-Paz J., Bennett E.J., Kay S.A., and Gendron J.M. (2018) Decoys untangle complicated redundancy and reveal targets of circadian clock F-box proteins. Plant Physiol. 177, 1170-1186.

Lee, J.Y., Wang, X., Cui, W., Sager, R., Modla, S., Czymmek, K., Zybaliov, B., van Wijk, K., Zhang, C., Lu, H., and Lakshmanan, V. (2011). A plasmodesmata-localized protein mediates crosstalk between cell-to-cell communication and innate immunity in Arabidopsis. Plant Cell 23, 3353-3373. 
Li, Z., Wang, X., Chen, J., Gao, J., Zhou, X., and Kuai, B. (2016) CCX1, a Putative Cation/Ca2+ Exchanger, Participates in Regulation of Reactive Oxygen Species Homeostasis and Leaf Senescence. Plant Cell Physiol 57, 2611-2619.

Li, Z., Variz, H., Chen Y., Liu S.L., and Aung K. (2020) Plasmodesmata-dependent intercellular movement of bacterial effectors. bioRxiv. doi: https://doi.org/10.110/2020.12.10.420240.

Liesche, J. (2017). Sucrose transporters and plasmodesmal regulation in passive phloem loading. J. Integr. Plant Biol. 59, 311-321.

Liesche, J. and Patrick J. (2017). An update on phloem transport: a simple bulk flow under complex regulation. F1000Res 6, 2096. doi: 10.12688/f1000research.12577.1.

Lucas, W.J., Ham, B.K., and Kim, J.Y. (2009). Plasmodesmata - bridging the gap between neighboring plant cells. Trends Cell Biol. 19, 495-503.

Lucas, W.J., Groover A., Lichtenberger R., Furuta K., Yadav, S.R., Helariutta, Y., He, X.Q., Fukuda, H., Kang, J., Brady, S.M., Patrick, J.W., Sperry, J., Yoshida, A., LópezMillán, A.F., Grusak, M.A., and Kachroo, P. (2013). The plant vascular system: Evolution, Development, and Functions. J. Integr. Plant Biol. 55, 294-388.

Nakagawa, T., Kurose, T., Hino, T., Tanaka, K., Kawamukai, M., Niwa, Y., Toyooka, K., Matsuoka, K., Jinbo, T., and Kimura, T. (2007). Development of series of gateway binary vectors, pGWBs, for realizing efficient construction of fusion genes for plant transformation. J. Biosci Bioeng. 104, 34-41.

Oparka K.J. and Turgeon R. (1999). Sieve elements and companion cells-traffic control centers of the phloem. Plant Cell 11, 739-750.

Sager R.E. and Lee J.Y. (2018). Plasmodesmata at a glance. J. Cell Sci. 131, jcs209346. doi: $10.1242 /$ jcs. 209346.

Schulz, A. (2015). Diffusion or bulk flow: how plasmodesmata facilitate pre-phloem transport of assimilates. J. Plant Res. 128, 49-61.

Srivastave A.C., Ganesan S., Ismail I.O., and Ayre B.G. (2008). Functional characterization of the Arabidopsis AtSUC2 Sucrose/H+ symporter by tissue-specific complementation reveals an essential role in phloem loading but not in long-distrance transport. Plant Physiol. 148, 200-211.

Thomas, C.L., Bayer, E.M., Ritzenthaler, C., Fernandez-Calvino, L., and Maule, A.J. (2008). Specific targeting of a plasmodesmal protein affecting cell-to-cell communication. PLoS biology 6, e7.

Tomczynska, I., Stumpe, M., Doan, T.G., and Mauch, F. (2020). A Phytophthora effector protein promotes symplastic cell-to-cell trafficking by physical interaction with plasmodesmata-localised callose synthases. New Phytol. 227, 1467-1478.

Wang, X., Robles Luna, G., Arighi, C.N., and Lee, J.Y. (2020). An evolutionarily conserved motif is required for Plasmodesmata-located protein 5 to regulate cell-to-cell movement. Commun. Biol. 3, 291.

Zhang, C., and Turgeon, R. (2018). Mechanisms of phloem loading. Cur. Opin. Plant Biol. 43, 71-75. 


\section{Figure legend}

Figure 1. The overexpression of PDLP5 and PDLP6 leads to stunted growth and starch overaccumulation. (a) Immunoblot analysis detects the expression of PDLP6-HF in four independent transgenic plants. An anti-Flag antibody was used to detect the expression of Flagfusion proteins. Rubisco was served as loading controls. (b) 3-week-old Arabidopsis plants were grown under a light intensity used for standard Arabidopsis growth $\left(110 \mu \mathrm{mol} \mathrm{m}^{-2} \mathrm{~s}^{-1}\right)$. (c) Starch accumulation phenotype of four independent transgenic lines expressing PDLP6-HF. The plants were grown under a light intensity used for standard Arabidopsis growth $\left(110 \mu \mathrm{mol} \mathrm{m}^{-2} \mathrm{~s}^{-1}\right)$ for three weeks. Plants were subjected for starch staining using Lugol's solution at the end of the night. (d) Starch staining of 5-week-old Arabidopsis plants. 4-week-old Arabidopsis plants were irradiated with a high light intensity $\left(200 \mu \mathrm{mol} \mathrm{m}^{-2} \mathrm{~s}^{-1}\right)$ for a week. Samples were collected at the end of the night for starch staining using Lugol's solution. All images were captured with the same magnification. (e) Histological sections of Arabidopsis leaves. Mature leaves of 5-week-old high light-treated plants were subjected to sectioning and staining with periodic acid/Schiff reagent, which stains polysaccharide in cell wall and starch grains in chloroplasts. Asterisks mark bundle sheath cells. Scale bars $=20 \mu \mathrm{m}$.

Figure 2. PDLP5 and PDLP6 express in and function at different cell types. (a) The cell typespecific expression of PDLP5-YFP and PDLP6-YFP. The fusion proteins were driven by their respective native promoter. Confocal images were captured from 2-week-old Arabidopsis seedlings. Green signals indicate the expression of the YFP fusion proteins in different cell types (top panel). Merged images of the signals from YFP and bright field are shown in the lower panel. White arrow heads indicate the expression of PDLP6-YFP at PD of vascular cells. Magenta arrow

421 heads indicate chlorophyll autofluorescence. Scale bars for epidermis and vasculature $=10 \mu \mathrm{m}$.

422 Scale bars for root $=50 \mu \mathrm{m}$. (b) Callose accumulation between leaf epidermal or mesophyll cells 423 in transgenic plants overexpressing HF-YFP (control), PDLP5-HF, or PDLP6-HF. Green signals 424 present aniline blue-stained callose at PD (top panel). Merged images of the signals from callose and bright field are shown in the lower panel. Scale bars $=10 \mu \mathrm{m}$. (c) Quantitative data show callose accumulation between epidermal cells and mesophyll cells. Asterisks indicate differences

427 that are statistically significant (Mann-Whitney UTest; $\mathrm{p}<0.0002$ ). n.s.: not significant. (d) Callose accumulation in roots of the transgenic plants. Green signals present callose accumulation in 
different root cell types (top panel). Scale bars $=50 \mu \mathrm{m}$. Merged images of bright field and fluorescence intensity profile (arbitrary unit: AU) of callose accumulation along the horizontal lines are shown in the lower panel. Numbers on the $\mathrm{X}$ axis indicate distance $(\mu \mathrm{m})$ across the region

432 analyzed.

Supplemental Figure 1. Characterization of p35S::PDLP5-HF and p35S::PDLP6-HF transgenic plants. (a) Immunoblot analysis detects the expression of PDLP5-HF in two independent transgenic plants. An anti-Flag antibody was used to detect the expression of Flagfusion proteins. Rubisco was served as loading controls. (b) Starch accumulation phenotype of two independent transgenic lines expressing PDLP5-HF. The plants were grown under a light intensity used for standard Arabidopsis growth $\left(110 \mu \mathrm{mol} \mathrm{m}^{-2} \mathrm{~s}^{-1}\right)$ for four weeks. Plants were subjected for starch staining using Lugol's solution at the end of the night. (c) Stunted growth and late flowering phenotypes of Arabidopsis transgenic plants expressing PDLP5-HF or PDLP6-HF. p35S::HF-YFP was served as a control. Images were taken from 5-week-old plants using the same magnification.

Supplemental Figure 2. Starch accumulation phenotype of Arabidopsis mutants and transgenic plants. (a) The plants were grown under a light intensity used for standard Arabidopsis growth (110 $\mu \mathrm{mol} \mathrm{m} \mathrm{m}^{-2} \mathrm{~s}^{-1}$; top panel) for four weeks. (b) For high light treatment, 4-week-old Arabidopsis plants were irradiated with a high light intensity $\left(200 \mu \mathrm{mol} \mathrm{m}^{-2} \mathrm{~s}^{-1}\right.$; lower panel $)$ for a

\section{Supplemental Figure 3. The promoter activities of Arabidopsis PDLP5 and PDLP6 genes.}

452 Histochemical GUS analysis of Arabidopsis transgenic plants expressing the reporter genes under

453 the control of the PDLP5 or PDLP6 gene promoter of Arabidopsis. Leaves of 4-week-old 454 Arabidopsis $T_{1}$ transgenic plants were subjected to GUS staining. Scale bar $=100 \mu \mathrm{m}$. Roots of 2455 week-old Arabidopsis $\mathrm{T}_{2}$ transgenic plants were subjected to GUS staining. Scale bar $=50 \mu \mathrm{m}$. 456 The numbers indicate the transgenic plants exhibit the shown GUS activity pattern out of the total 457 independent transgenic plants analyzed. 
459 Supplemental Figure 4. Callose accumulation in roots of Arabidopsis transgenic plants 460 overexpressing HF-YFP, PDLP5-HF, or PDLP6-HF. Semi-quantitative evaluation of the

461 relative level of aniline blue-stained callose in root cells was performed by analyzing the signal

462 intensity across different root cell types. Confocal images were captured from the maturation zone 463 of 10-day old seedlings. 25 individual transgenic plants were analyzed for each genotype and 464 fluorescence intensity profiles (arbitrary unit: AU) of callose accumulation were combined within 465 the genotype. Numbers on the $\mathrm{X}$ axis indicate distance $(\mu \mathrm{m})$ across the region analyzed.

467 Supplemental Figure 5. Ubiquitous expression of PDLP5-YFP and PDLP6-YFP fusion 468 proteins in p35S::PDLP5-YFP and p35S::PDLP6-YFP transgenic plants. The expression of 469 the fusion proteins was detected in epidermal cells, mesophyll cells, vascular cells in leaves, and 470 most cell types in roots. Confocal images were captured from 2-week-old Arabidopsis seedlings.

471 Scale bars for epidermis, mesophyll, and vasculature $=10 \mu \mathrm{m}$. Scale bars for root $=50 \mu \mathrm{m}$.

472

473 Supplemental Table 1. Primers used for cloning in this study. 
a

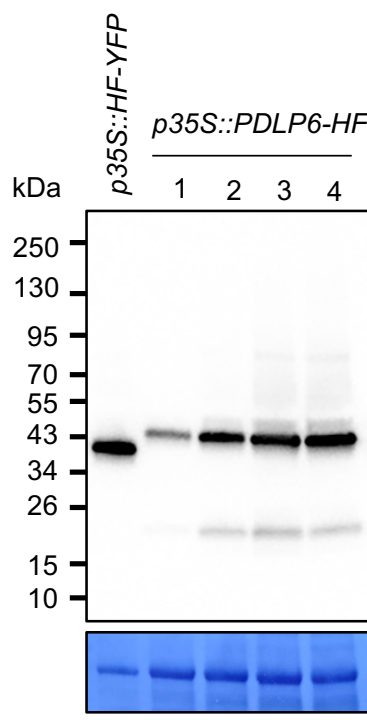

b

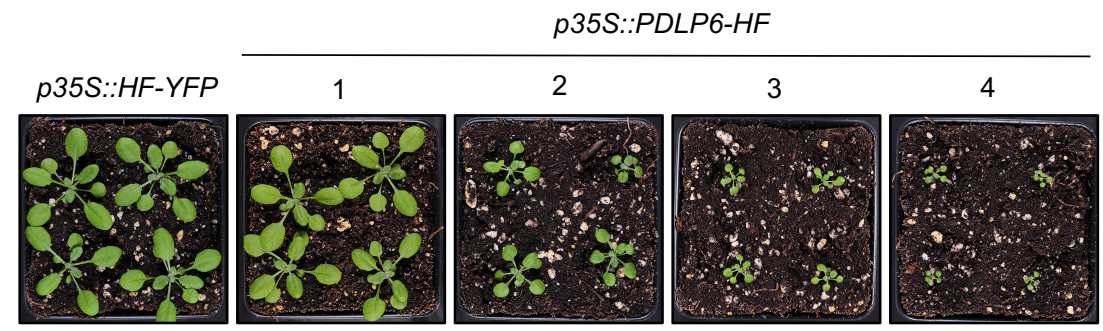

C

p35S::PDLP6-HF

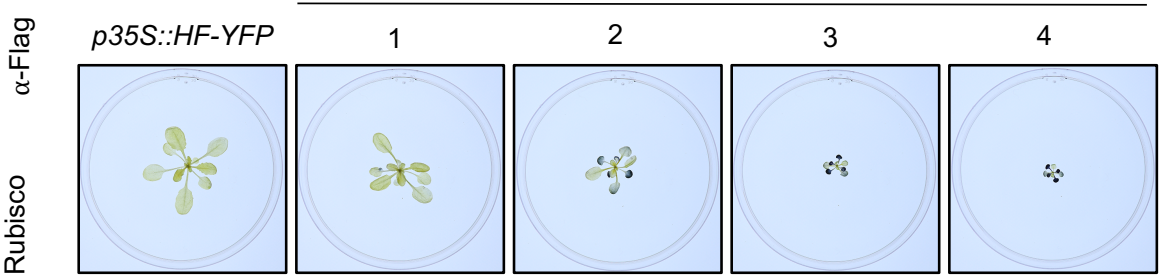

d

Col-0 cher1-4

sweet $11 ; 12$
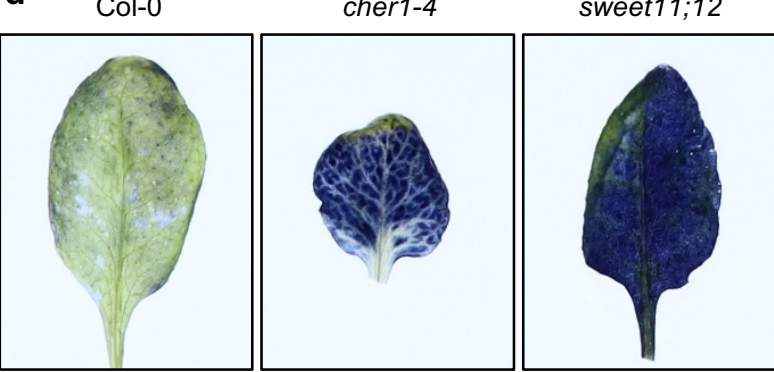

p35S::HF-YFP

p35S::PDLP5-HF p35S::PDLP6-HF
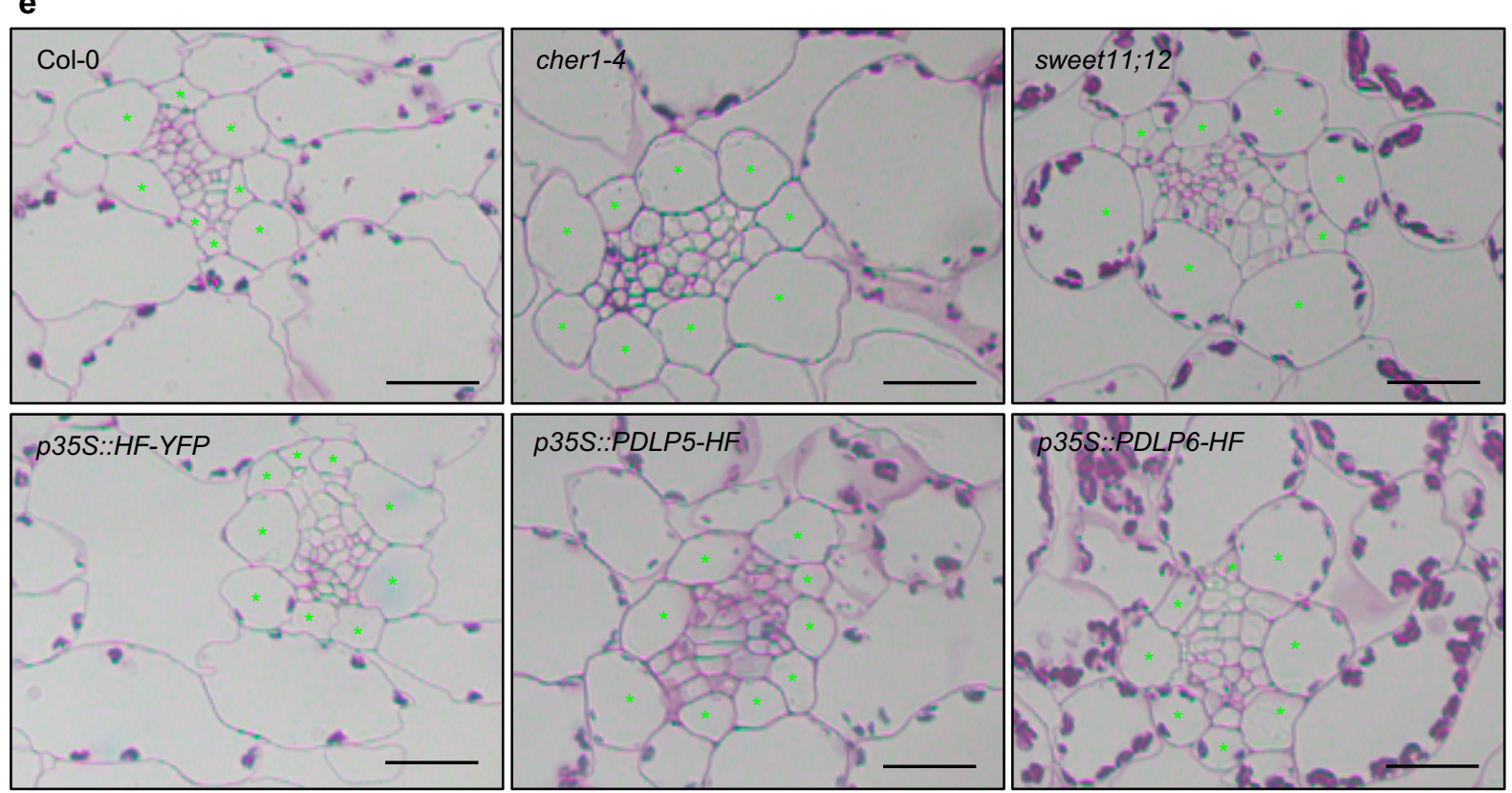

Figure 1. The overexpression of PDLP5 and PDLP6 leads to stunted growth and starch overaccumulation. (a) Immunoblot analysis detects the expression of PDLP6-HF in four independent transgenic plants. An anti-Flag antibody was used to detect the expression of Flag-fusion proteins. Rubisco was served as loading controls. (b) 3-week-old Arabidopsis plants were grown under a light intensity used for standard Arabidopsis growth $\left(110 \mu \mathrm{mol} \mathrm{m} \mathrm{m}^{-2} \mathrm{~s}^{-1}\right)$. (c) Starch accumulation phenotype of four independent transgenic lines expressing PDLP6-HF. The plants were grown under a light intensity used for standard Arabidopsis growth $\left(110 \mu \mathrm{mol} \mathrm{m} \mathrm{m}^{-2} \mathrm{~s}^{-1}\right)$ for three weeks. Plants were subjected for starch staining using Lugol's solution at the end of the night. (d) Starch staining of 5-week-old Arabidopsis plants. 4-week-old Arabidopsis plants were irradiated with a high light intensity $\left(200 \mu \mathrm{mol} \mathrm{m}^{-2} \mathrm{~s}^{-1}\right)$ for a week. Samples were collected at the end of the night for starch staining using Lugol's solution. All images were captured with the same magnification. (e) Histological sections of Arabidopsis leaves. Mature leaves of 5-week-old high light-treated plants were subjected to sectioning and staining with periodic acid/Schiff reagent, which stains polysaccharide in cell wall and starch grains in chloroplasts. Asterisks mark bundle sheath cells. Scale bars $=20 \mu \mathrm{m}$. 
a

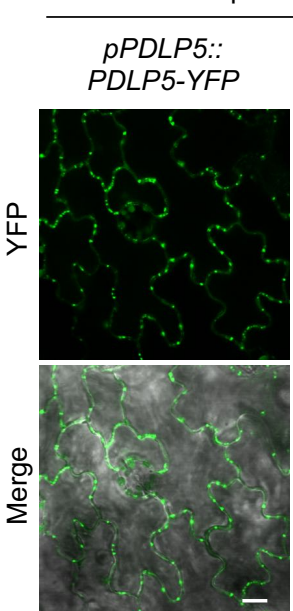

Epidermis

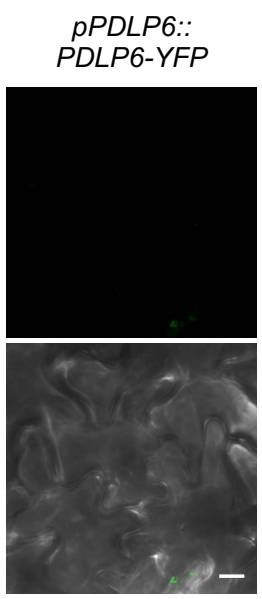

Epidermis
p35S::PDLP6-HF

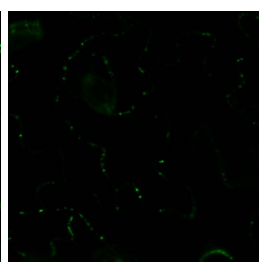

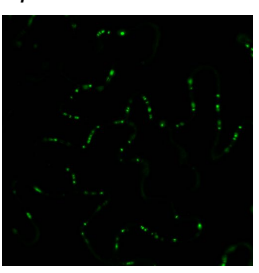
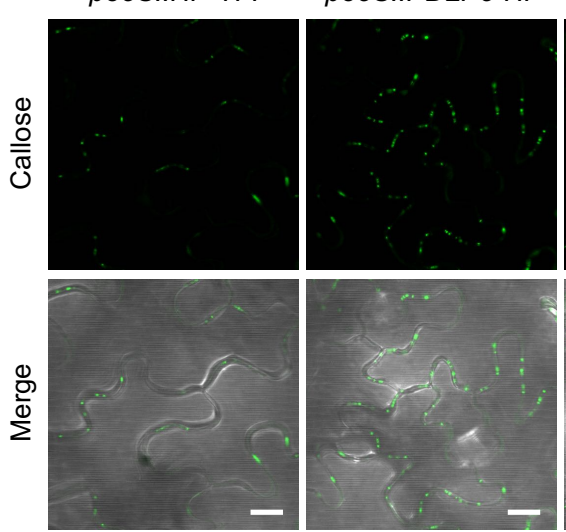

Vasculature

pPDLP5::
PDLP5-YFP
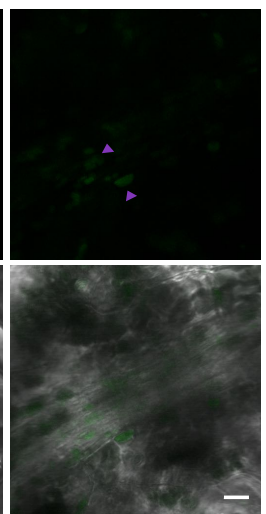

pPDLP6::
PDLP6-YFP

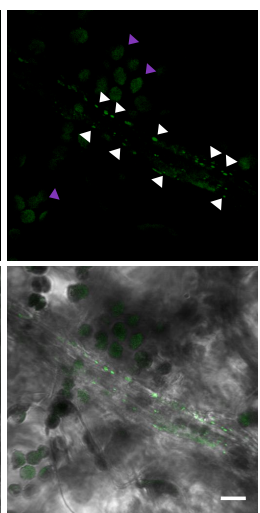

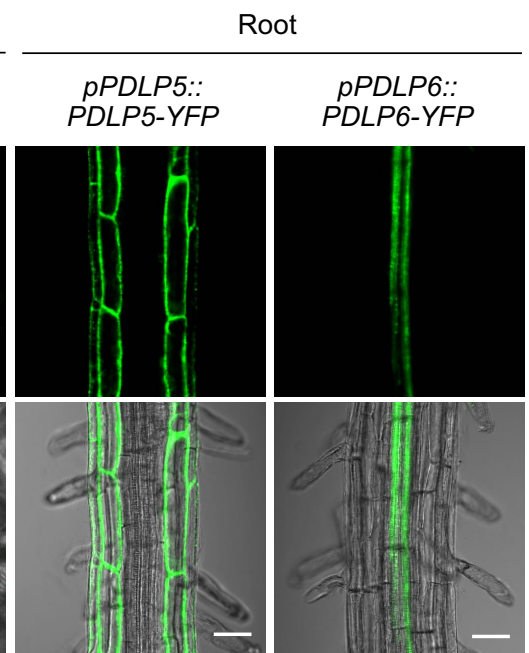

Mesophyll
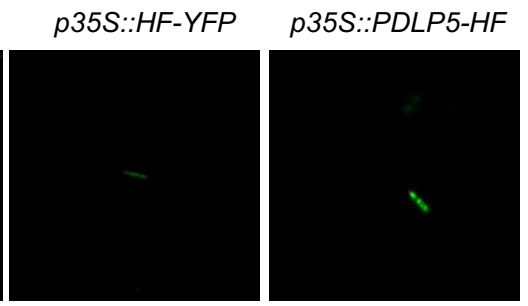

p35S::PDLP6-HF
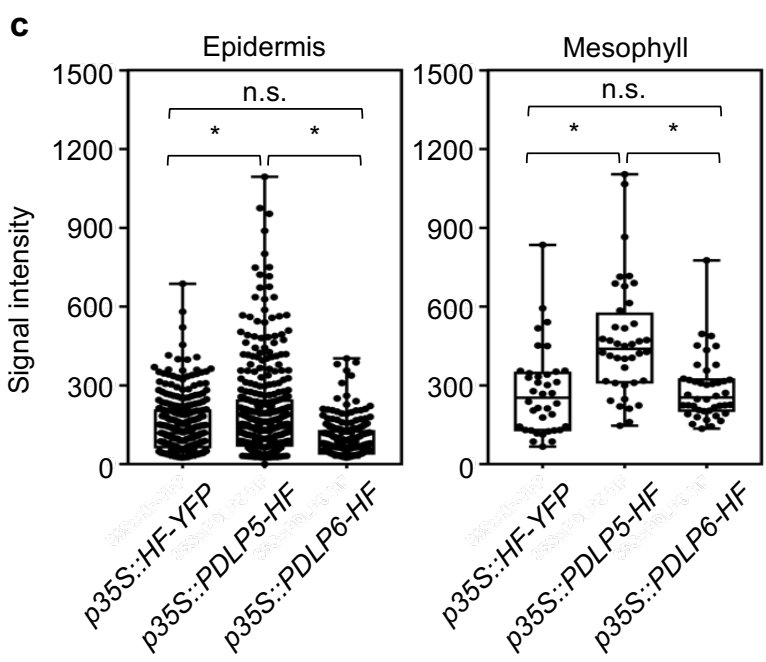

d

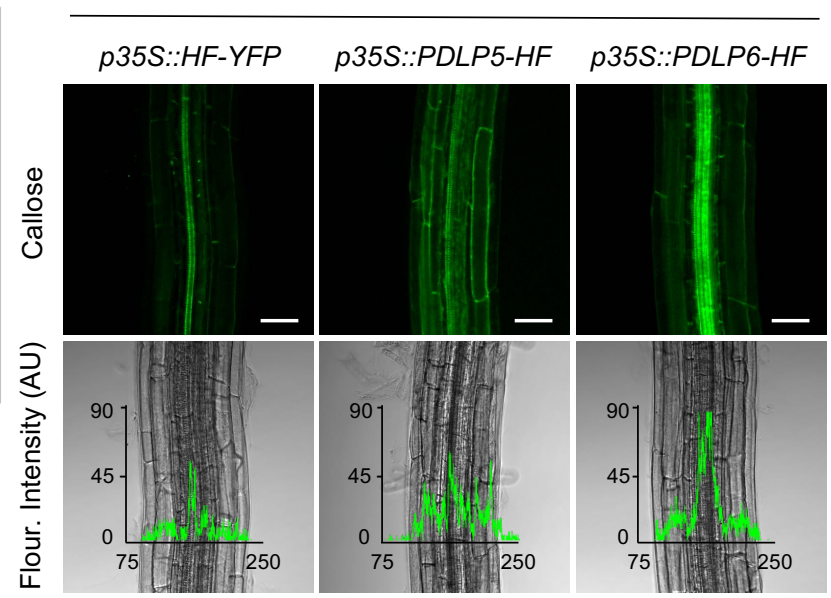

Figure 2. PDLP5 and PDLP6 express in and function at different cell types. (a) The cell type-specific expression of PDLP5YFP and PDLP6-YFP. The fusion proteins were driven by their respective native promoter. Confocal images were captured from 2-week-old Arabidopsis seedlings. Green signals indicate the expression of the YFP fusion proteins in different cell types (top panel). Merged images of the signals from YFP and bright field are shown in the lower panel. White arrow heads indicate the expression of PDLP6-YFP at PD of vascular cells. Magenta arrow heads indicate chlorophyll autofluorescence. Scale bars for epidermis and vasculature $=10 \mu \mathrm{m}$. Scale bars for root $=50 \mu \mathrm{m}$. (b) Callose accumulation between leaf epidermal or mesophyll cells in transgenic plants overexpressing HF-YFP (control), PDLP5-HF, or PDLP6-HF. Green signals present aniline blue-stained callose at PD (top panel). Merged images of the signals from callose and bright field are shown in the lower panel. Scale bars = $10 \mu \mathrm{m}$. (c) Quantitative data show callose accumulation between epidermal cells and mesophyll cells. Asterisks indicate differences that are statistically significant (Mann-Whitney $U$ Test; $p<0.0002$ ). n.s.: not significant. (d) Callose accumulation in roots of the transgenic plants. Green signals present callose accumulation in different root cell types (top panel). Scale bars $=50$ $\mu \mathrm{m}$. Merged images of bright field and fluorescence intensity profile (arbitrary unit: AU) of callose accumulation along the horizontal lines are shown in the lower panel. Numbers on the $X$ axis indicate distance $(\mu \mathrm{m})$ across the region analyzed. 